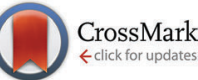

Cite this: Mol. BioSyst., 2015, 11, 2907

Received 22nd May 2015, Accepted 31st July 2015

DOI: $10.1039 / \mathrm{c} 5 \mathrm{mb} 00352 \mathrm{k}$

www.rsc.org/molecularbiosystems

\title{
Computational predictions of corroles as a class of Hsp90 inhibitors $\dagger$
}

\author{
Ruijie D. Teo, $\ddagger^{\mathrm{ab}}$ Sijia S. Dong, $\ddagger^{\mathrm{ab}}$ Zeev Gross, ${ }^{c}$ Harry B. Gray ${ }^{\mathrm{b}}$ and \\ William A. Goddard III*ab
}

\begin{abstract}
Corroles have been shown experimentally to cause cell cycle arrest, and there is some evidence that this might be attributed to an inhibitory effect of corroles on Heat shock protein 90 (Hsp90), which is known to play a vital role in cancer cell proliferation. In this study, we used molecular dynamics to examine the interaction of gallium corroles with Hsp90, and found that they can bind preferentially to the ATP-binding $\mathrm{N}$-terminal site. We also found that structural variations of the corrole ring can influence the binding energies and affinities of the corrole to Hsp90. We predict that both the biscarboxylated corrole (4-Ga) and a proposed 3,17-bis-sulfonated corrole (7-Ga) are promising alternatives to $\mathrm{Ga}(\mathrm{III})$ 5,10,15-tris(pentafluorophenyl)-2,17-bis(sulfonic acid)-corrole (1-Ga) as anti-cancer agents.
\end{abstract}

\section{Introduction}

Corroles, and in particular the metal chelates of $5,10,15$ tris(pentafluorophenyl)-2,17-bis(sulfonic acid)-corrole 1- $\mathrm{H}_{3}, 1-\mathrm{M}$ (Scheme 1), have become increasingly important in recent years due to their utility in medical applications. The iron and manganese complexes display excellent catalytic activity for decomposition of the main reactive oxygen species (ROS) and reactive nitrogen species (RNS) that are implicated in numerous diseases. Many beneficial effects have been disclosed for 1-Fe in model systems of diabetes, ${ }^{1}$ neurodegeneration, ${ }^{2}$ optic neuropathy, ${ }^{3}$ and atherosclerosis. ${ }^{4} 1-\mathrm{Mn}$ and similar manganese corroles are most effective for the attenutation of molecular and intracellular nitration. 1-Sb is able to photodynamically inactivate mold fungi spores. ${ }^{5}$ 1-Al and 1-Ga are highly fluorescent, ${ }^{6}$ and 1-Au and 1-Ga have been shown to exhibit cytotoxicity in a number of cancer cell lines. ${ }^{7}$

These amphiphilic bis-sulfonated metallocorroles interact with a variety of proteins, including albumin ${ }^{8}$ and transferrin. ${ }^{9}$ Recently, metallocorroles have been shown to bind tightly to low-density lipoprotein (LDL) and a sub-fraction of high-density lipoprotein (HDL) upon mixing with human serum to protect them from oxidative/nitrative damage. ${ }^{10}$

\footnotetext{
${ }^{a}$ Materials and Process Simulation Center, California Institute of Technology, Pasadena, California 91125, USA. E-mail:wag@wag.caltech.edu

${ }^{b}$ Division of Chemistry and Chemical Engineering, California Institute of Technology, Pasadena, California 91125, USA

${ }^{c}$ Schulich Faculty of Chemistry, Technion-Israel Institute of Technology, Haifa 32000, Israel

$\dagger$ Electronic supplementary information (ESI) available. See DOI: 10.1039/ c5mb00352k

\$ These authors contributed equally to this work.
}

1-Ga spontaneously assembles with a heregulin-modified protein directed at the human epidermal growth factor receptor (HER), leading to targeted tumor cell death in a mouse model. This can be observed by the intense fluorescence of the corrole rendering it suitable for both cellular and whole animal optical imaging. ${ }^{11,12}$ Although there have been large advances in<smiles>O=Cc1c(F)c(F)c(F)c(F)c1F</smiles>

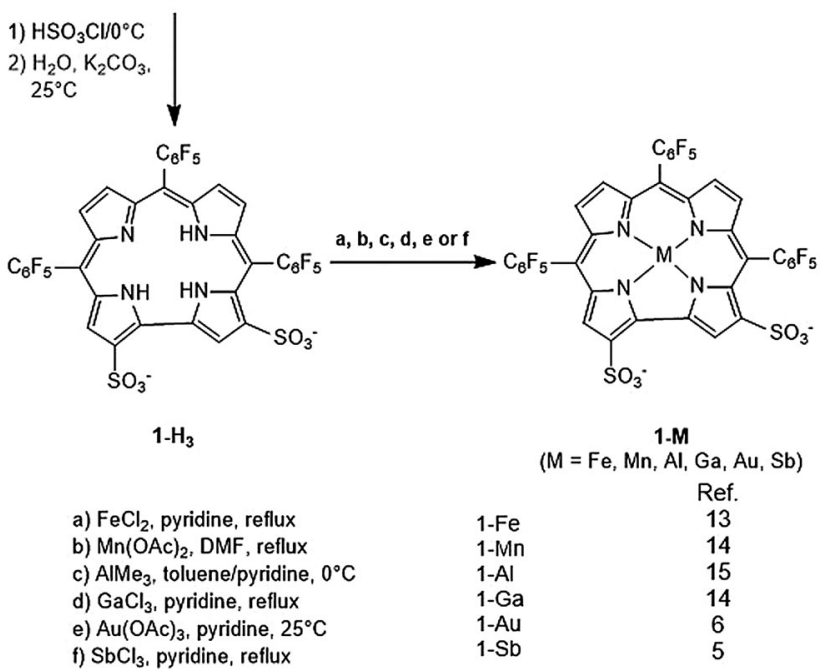

Scheme 1 Synthesis of cytotoxic metallocorroles 1-M. 
elucidating the cancer cell-killing mechanism by $1-\mathrm{Ga},{ }^{16,17}$ both free and protein-conjugated, many open questions about its intracellular behavior remain unanswered.

One such class of intracellular proteins that have been implicated in cancer is heat shock proteins (Hsp). ${ }^{18}$ Hsp are ubiquitous molecular chaperones distributed from bacteria to animal cells and essential mediators of signal-transduction and cell cycle progression in prokaryotes and eukaryotes. ${ }^{19}$ In particular, Hsp90, a subclass of Hsp that is often implicated in tumorigenesis and tumor progression, aids in a wide range of intracellular processes - protein assembly, trafficking, folding, degradation, and neurodegenerative diseases. ${ }^{20,21}$ Since Hsp90 has a concentration 2-10 times higher in tumors than normal cells, it has been recognized as a potential target for cancer. ${ }^{22}$ Indeed the development of Hsp90 inhibitors has been rapid - 17 agents had entered clinical trials in 2012. ${ }^{23}$ Potent Hsp90 inhibitors such as geldanamycin and radicicol (Fig. 1) block the ATP binding/hydrolysis site at the N-terminal nucleotidebinding pocket, inhibiting its function, depleting oncogenic clients, and engendering antitumor activity. ${ }^{23-25}$ Studies in both human breast cancer cells and dopaminergic neural cells showed that 19-substituted benzoquinone ansamycin (BQA) macrocycles are effective Hsp90 inhibitors while possessing markedly reduced toxicity to normal endothelial and epithelial cells than their parent quinones. ${ }^{26}$

Previous work showed that tubocapsenolide A inhibits activity of the Hsp90-Hsp70 chaperone complex, leading to destabilization of Hsp90 client proteins and subsequent cell cycle arrest and apoptosis in MDA-MB-231 cells. ${ }^{27}$ In 2012, Termini and coworkers found that 1-Ga causes mitotic cell cycle arrest in the same MDA-MB-231 cell line. ${ }^{28}$ Most recently, protoporphyrin IX (PPIX) treatment was purported to inhibit the chaperone activity of Hsp90, accelerating protein degradation of HIF- $1 \alpha^{29}$ These developments, coupled with the structural similarity of PPIX and 1-Ga, led us to hypothesize that 1-Ga's tumor killing ability is from its inhibition of Hsp90. In this study, we predicted the binding site for 1-Ga and its derivatives (Fig. 2) to Hsp90 which we compared with the predicted binding sites of PPIX and ATP with Hsp90. Then we carried out molecular dynamics (MD) simulation of the docked complexes. These studies led to corrole-Hsp90 interactions that support our hypothesis suggesting how corroles might inhibit Hsp90. These findings also provide insight into designing potent corrole-based anticancer drugs.

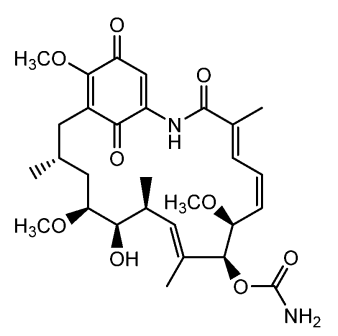

1

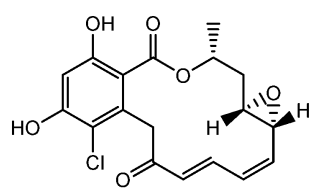

2
Fig. 1 Structures of geldanamycin 1 and radicicol 2.
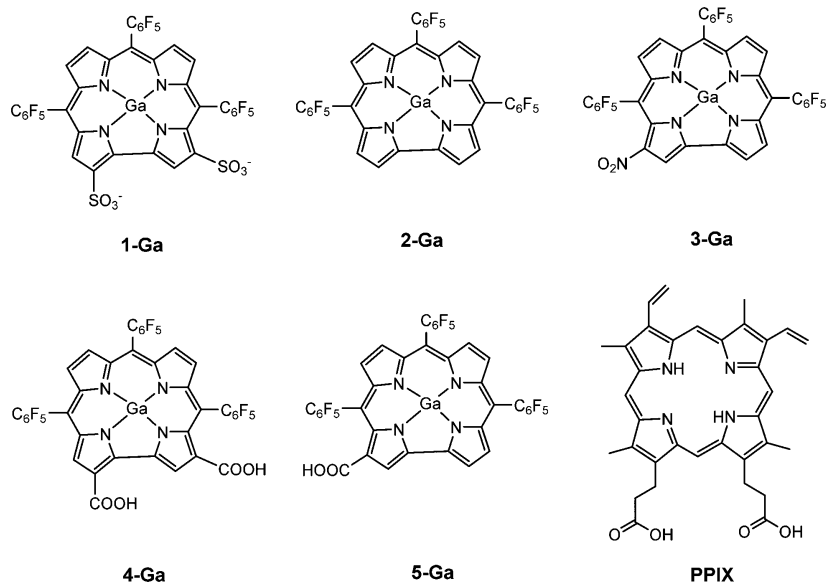

Fig. 2 Compounds investigated in this study - gallium corrole analogues 1-Ga to 5-Ga, and PPIX (with the exception of ATP).

\section{Procedures}

\section{Preparation of the protein and ligands}

1- to 5-Ga were constructed using the crystal structure of a structurally similar gold corrole. ${ }^{30}$ Starting with the co-crystal structure of Hsp90 with geldanamycin (protein data bank (PDB) code: 1YET) we removed the ligand and all crystallographic water molecules. The structure for PPIX was obtained from a co-crystal structure of human ferrochelatase with PPIX (PDB code: $2 \mathrm{HRE}$ ) while the structure of ATP was obtained from the crystal structure of ATP-bound monomeric actin (PDB code: 2HF4). All ligands tested were then geometrically optimized with quantum mechanics [the B3LYP-D3 level of density functional theory (DFT) that includes D3 corrections for van der Waals attraction] using the $6-31 \mathrm{G}^{* *++}$ basis set for the non-metal atoms and the LACV3P $\mathrm{P}^{31,32}$ basis set for the metal and including the Poisson-Boltzmann model for implicit solvation by water, as implemented in the Jaguar 8.0 package. ${ }^{33}$ We obtained the Mulliken charges from these DFT calculations for use in docking the ligands to the protein structure of Hsp90.

\section{Molecular docking}

Molecular docking studies were performed using our GenDock ${ }^{34,35}$ procedure using the DREIDING force field (FF).$^{36}$ GenDock has been shown to be able to predict protein-ligand binding sites and energy consistent with experimental findings for a number of cases including $\mathrm{G}$ protein-coupled receptors ${ }^{34,37}$ and cyclindependent kinase $5 .^{38}$ We first generated all possible ligandbinding positions in the alanized protein (the protein with all hydrophobic residues mutated to alanine) by filling the binding regions with spheres that are classified into boxes with 10 A sides. Then we used $\mathrm{PyMOL}^{39}$ to choose spheres in the N-terminal binding pocket of $\mathrm{Hsp} 90^{40}$ for docking. For each ligand, we screened 5 million poses without energy evaluation and kept the 98-109 poses that could fit into the chosen binding pocket. The poses were clustered into 3 families by RMSD and all poses were maintained. We then de-alanized these selected poses using SCREAM. Then we neutralized all charged residues in the 
protein except for Lys58 and Lys112 before geometry optimization of the poses, as both Lys58 and Lys112 are important mediators in ligand-Hsp90 interactions. ${ }^{41}$

\section{Molecular dynamics}

Starting with the docked structures, we inserted the ligandprotein into an equilibrated water box and carried out molecular dynamics (MD) calculations to relax the ligand-protein complex while allowing water to diffuse throughout the complex. These calculations used Amber force field with the NAMD 2.9 ${ }^{42} \mathrm{MD}$ software. We carried out $10 \mathrm{~ns}$ of MD at $310 \mathrm{~K}$ to select the best 1-Ga-Hsp90 complex from the docking studies. Our procedure was to use $\mathrm{VMD}^{43}$ to build an equilibrated orthorhombic water box that included the ligand-protein complex at the center and extended $10 \AA$ beyond the protein in all 6 directions. Then we eliminated all water molecules within $5 \AA$ of the complex. Using tleap from AmberTools11, ${ }^{44}$ we added sufficient $\mathrm{Cl}^{-}$and $\mathrm{Na}^{+}$ ions to neutralize the complex and to give a physiological salt concentration of $0.9 \% \mathrm{w} / \mathrm{v}$. The final system has 29996 atoms. Then we minimized the solvent for 5000 steps with the protein and ligand fixed, followed by $1 \mathrm{~ns}$ of NPT dynamics of the solvent using a bath temperature of $310 \mathrm{~K}$ and a pressure of $1 \mathrm{~atm}$. We then conjugate gradient minimized the whole resulting system for 5000 steps, followed by 10 ns NPT dynamics for the whole system at $310 \mathrm{~K}$ and $1 \mathrm{~atm}$. We used the Nosé-Hoover Langevin piston pressure control, with a damping coefficient of $5 \mathrm{ps}^{-1}$. The particle mesh Ewald (PME) method was applied for electrostatic interactions. Finally, the analysis of MD trajectories were performed using VMD.

\section{Corrole Amber parameters for MD}

Since Amber does not have a force field for corroles, we introduced new atom types and approximated the force constants from a published set of cobalt corrole parameters ${ }^{45}$ as well as GAFF. ${ }^{46}$ The well depth for Ga nonbonding interaction was taken from UFF. ${ }^{47}$ For the equilibrium bond distances and angles, we used the average bond distances and angles from the structure optimized using DFT (B3LYP-D3/LACV3P for Ga and B3LYP-D3/6-31G $\mathrm{G}^{*}++$ for all other atoms). These modified force field parameters are in Tables S1-S6 of the ESI. $\dagger$

\section{Results and discussion}

Of all the gallium corroles studied (Table 1), we find that 1-Ga has the strongest bonding to Hsp90 [based on the unified-cavity energy (ucav) and the snap binding energy (snapbe)]. 1-Ga also has the largest number of interactions of favourable interactions as indicated in Table 1. We find that the sulfonate groups of 1-Ga both make salt-bridges with the positively-charged amino group of Lys112, while Lys58 'hangs' from the top to form a cation-pi interaction with the conjugated corrole $\pi$-system (Fig. 3a). Most interesting is that we find an uncommon anion $-\pi$ interaction between the negatively-charged carboxylate group of Asp102 and the electron-poor $-\mathrm{C}_{6} \mathrm{~F}_{5}$ group. We also find interactions for the docking of other gallium corrole analogues, including 2-Ga, 3-Ga, and 5-Ga (Fig. 3). We predict that 4-Ga is bonded nearly as strongly as $1-\mathrm{Ga}$, but that $2-\mathrm{Ga}$ and $3-\mathrm{Ga}$ bind much more weakly (due to the lack of hydrophobic groups).

Table 1 Docking results of the best poses of 1-5-Ga, PPIX, and ATP to Hsp90. Here snapbe and scored ucav are two measure of the bond strength. Note that negative numbers for the binding energy indicate stronger bonds. The atom labels of the ligands are shown in Fig. S1 of the ESI

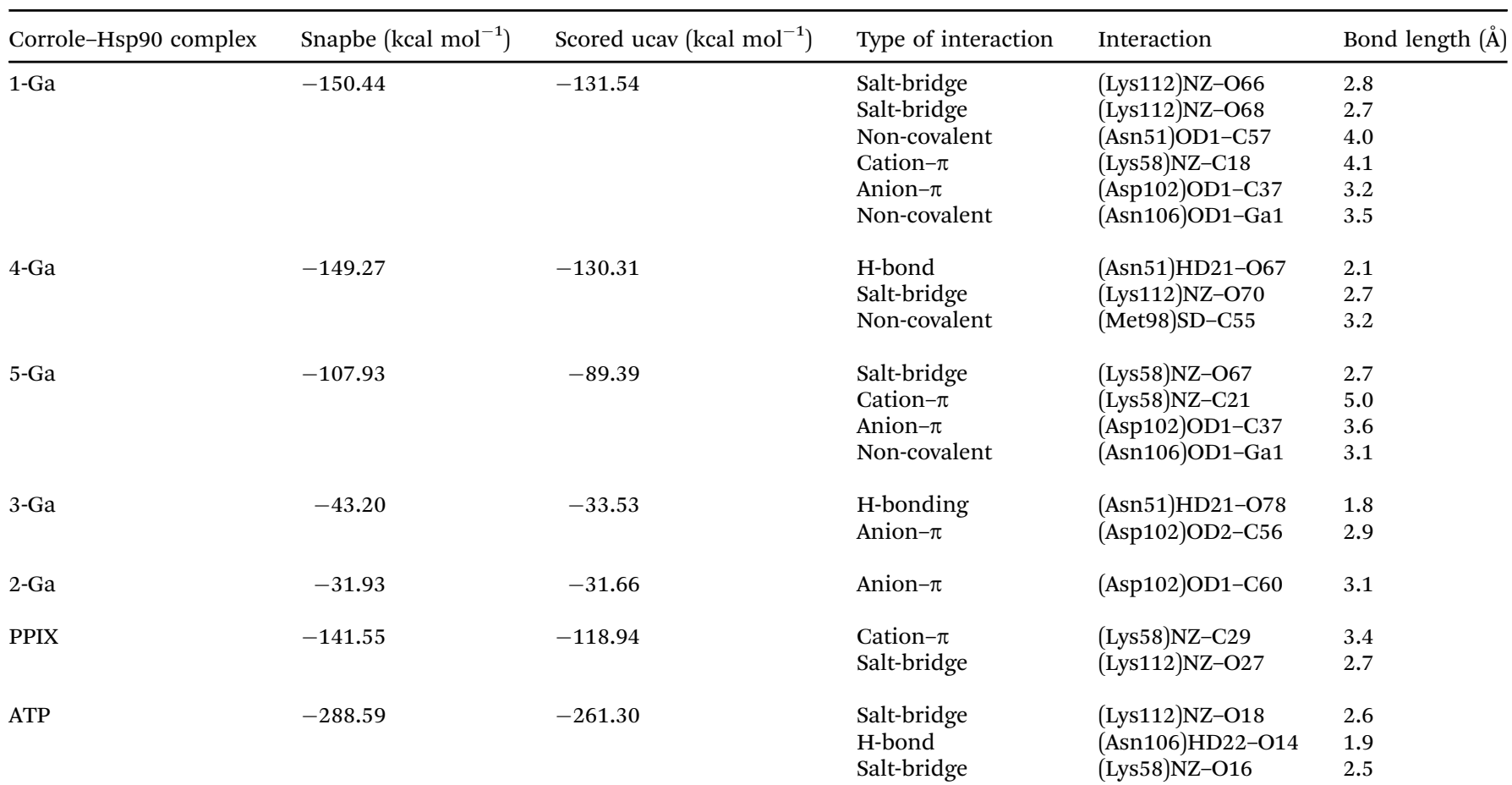



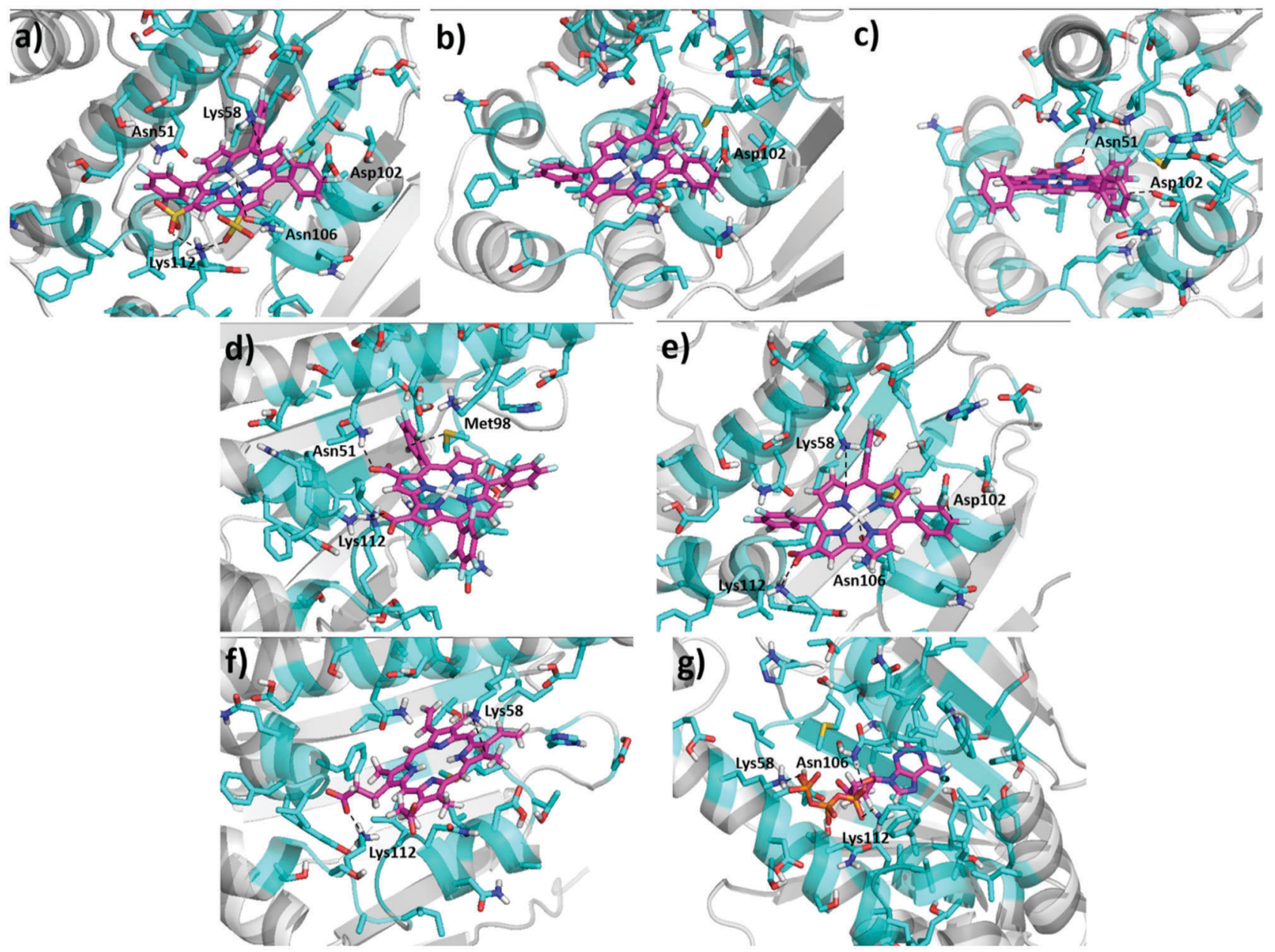

Fig. 3 Predicted ligand-Hsp90 structures at the N-terminal binding pocket of Hsp90 - (a) 1-Ga, (b) 2-Ga, (c) 3-Ga, (d) 4-Ga, (e) 5-Ga, (f) PPIX, and (g) ATP. Dotted lines represent possible interactions and binding modes, and atoms are classified by color according to type. The final pdb files for the coordinates of $1-\mathrm{Ga}-\mathrm{Hsp} 90$ and $4-\mathrm{Ga}-\mathrm{Hsp90}$ are listed in the ESI. $\dagger$

Also observed are axial interactions (Fig. 3a and e) as expected between the Lewis acidic $\mathrm{Ga}(\mathrm{III})$ and the amide group of Asn106.

DFT calculations were also performed to validate the $\mathrm{FF}$ calculations of these interactions (Fig. S2a and b, ESI $\dagger$ ). Comparing the predicted binding energies among 1- to 5-Ga, PPIX and ATP provides insight into designing of new Hsp90 inhibitors. We find that it is essential for the ligand to have three hydrophilic groups to achieve maximal binding at the $\mathrm{N}$-terminal site, which is the ATP binding site and relatively polar. ${ }^{40}$ For comparison we docked ATP to the N-terminal binding pocket, leading to interactions (Fig. $3 \mathrm{~g}$ ) consistent with those in the X-ray structure (PDB code: 1AM1). Since ATP is much smaller than the corroles and has more hydrophilic groups, we find that it binds more strongly than the other ligands investigated here.

Despite our prediction that ATP binds more strongly to Hsp90 than PPIX and 1- to 5-Ga, experiments show that PPIX and hemin directly impede the activation of hypoxia-inducible factor $1 \alpha$ (HIF-1 $\alpha$ ) by inhibiting Hsp90. ${ }^{29,48}$ The molecular details of the interactions between PPIX and Hsp90 have been addressed previously by computational docking studies. ${ }^{29}$ It was predicted that the conjugated aromatic system of porphyrin ring could make cation $-\pi$ interactions with Lys58, while one of the propionates form a hydrogen bond with Lys112. Indeed, our docking predictions confirm the purported cation- $\pi$ interaction of Lys58 with the porphyrin, and the direct salt-bridge interaction of Lys112 with one of the carboxylate groups (Fig. 3f). We also predict that the Hsp90 binding energy of 1-Ga and 4-Ga is stronger than that of PPIX, suggesting that 1-Ga and 4-Ga might be better Hsp90 inhibitors than PPIX.

To confirm our docking results, we carried out $10 \mathrm{~ns}$ of MD simulation on the docked complex 1-Ga-Hsp90. We consider that $10 \mathrm{~ns}$ is sufficient for our analyses because the RMSD changes of the Hsp90 backbone change are little during the last 5 ns (Fig. S3, ESI $\dagger$ ). The key interactions of 1-Ga with Hsp90 (Table 1) were analysed over the $10 \mathrm{~ns}$ as shown in Fig. 4. We find that these important interactions are relatively stable during the MD simulation (Fig. 5), with expected changes as water diffuses into the binding site. At the beginning of the MD simulation, Lys112 seems to be drawn slightly towards proximal groups (Gly135 and Asn106) probably because of the 

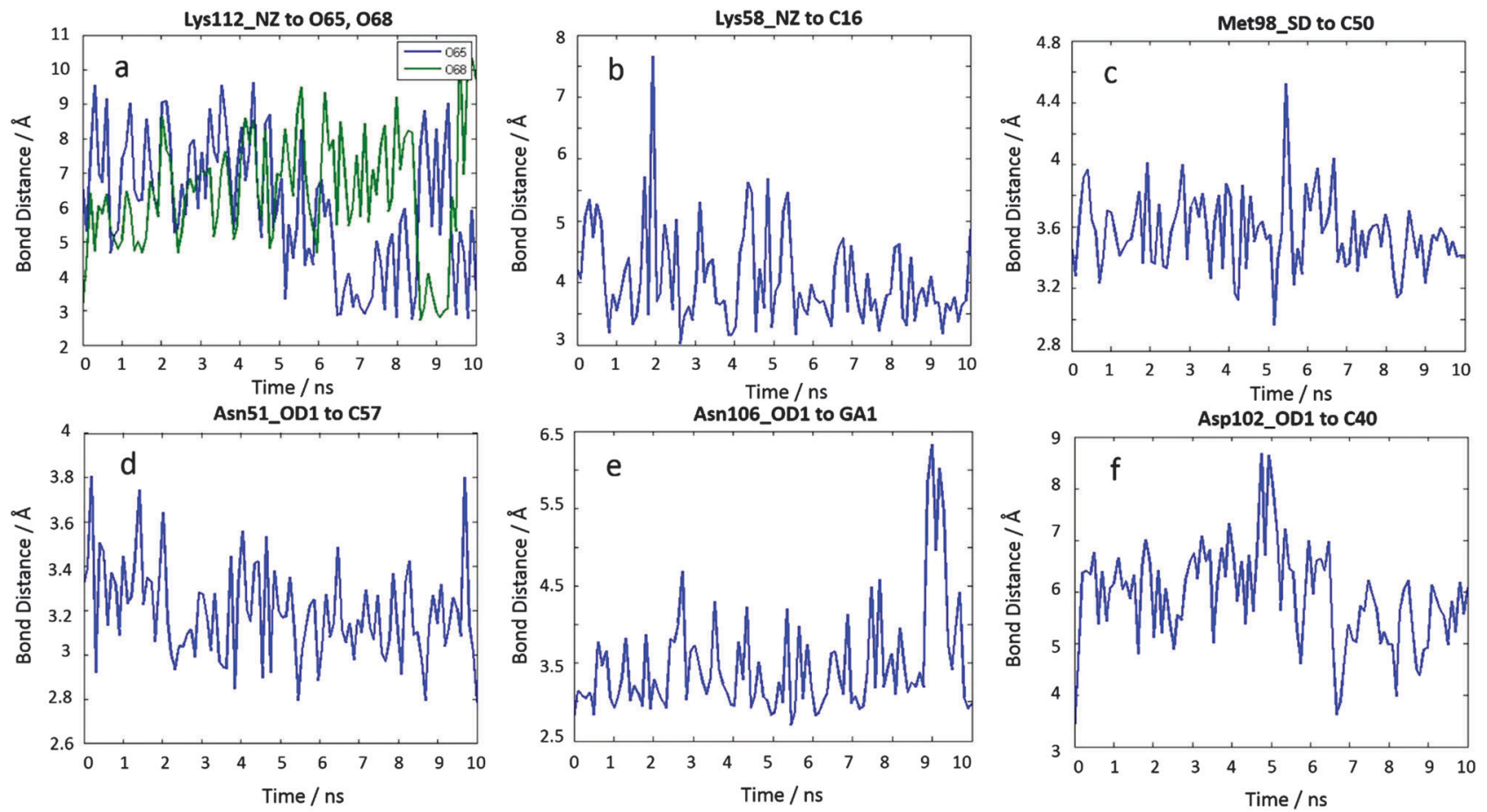

Fig. $410 \mathrm{~ns} \mathrm{MD}$ results of the interaction distances of 1-Ga to Hsp90 starting from the docking pose c4644. (a) Lys112 NZ to O65, O68, (b) Lys58 NZ to C16, (c) Met98 SD to C50, (d) Asn51 OD1 to C57, (e) Asn106 OD1 to GA1, and (f) Asp102 OD1 to C40.

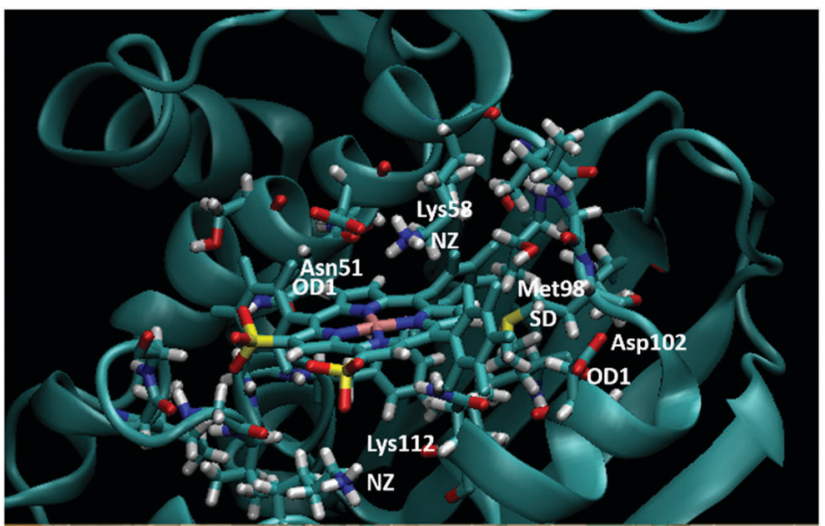

Fig. 5 Final frame of the MD for 1-Ga/Hsp90 after 10 ns MD. The starting pose is shown in Fig. S3 (ESI†). Residues important to binding are highlighted (color code: red $=\mathrm{O}$, blue $=\mathrm{N}$, white $=\mathrm{H}$, yellow $=\mathrm{S}$, pink $=\mathrm{Ga}$ ).

initial interactions with the water. However, after $5 \mathrm{~ns}$, the saltbridge interaction between the sulfonate group of 1-Ga and the protonated nitrogen of Lys112 eventually dominates and is maintained throughout (Fig. 4a). The non-covalent (Asn51 and $-\mathrm{C}_{6} \mathrm{~F}_{5}$ ring) and cation- $\pi$ interaction (Lys58 and corrole) are maintained throughout, while it is noted that the anion- $\pi$ interaction between OD1 of Asp102 and the $-\mathrm{C}_{6} \mathrm{~F}_{5}$ ring is present but weak. The axial interaction between the gallium center and the amide group of Asn106 is maintained throughout with only some aberrations at 9 ns (Fig. 4e). In addition, a sulfur $-\pi$ interaction ( $-\mathrm{S}$ and $-\mathrm{C}_{6} \mathrm{~F}_{5}$ ) is observed (Fig. $4 \mathrm{c}$ ). The pharmacophore of 1-Ga with Hsp90 is shown in Fig. 6.

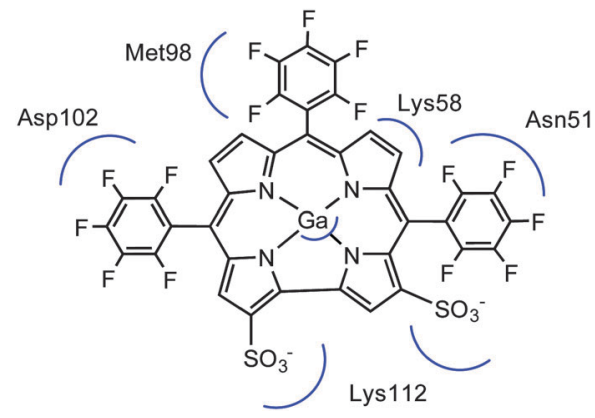

Fig. 6 Pharmacophore of 1-Ga to Hsp90. The Ga center interacts with Asn106 (not shown).

From the above analysis, we hypothesized that a ligand that could form two salt bridges between the corrole and Hsp90 (Lys58 and Lys112) might lead to stronger bonding than 1-Ga. Since the two sulfonate groups in 1-Ga are not far apart enough to form salt bridges with both Lys58 and Lys112 simultaneously,
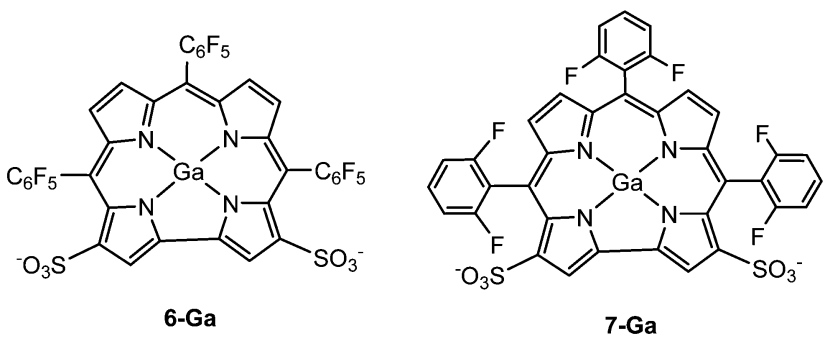

Fig. 7 Structures of 6-Ga and 7-Ga. 


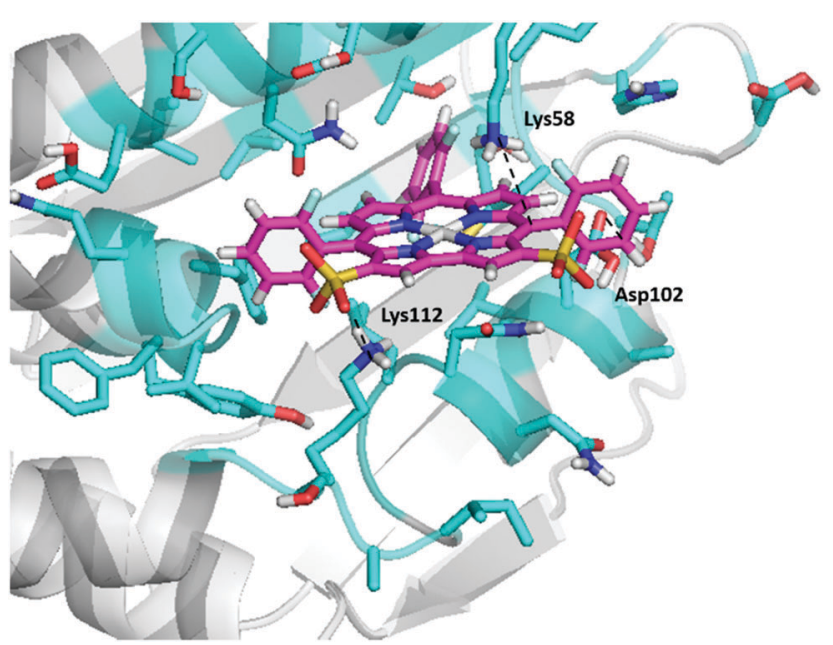

Fig. 8 Docking of 7-Ga to the N-terminal binding pocket of Hsp90. The final pdb files for the coordinates of 7-Ga-Hsp90 are listed in the ESI. $\dagger$ we proposed 6-Ga and 7-Ga (Fig. 7) and carried out both docking and $10 \mathrm{~ns}$ of $\mathrm{MD}$ simulations to test their binding ability to Hsp90. The MD results for 6-Ga are listed in Fig. S5-S8 (ESI $\dagger$ ). The binding mode stabilizes after 7 ns (Fig. S5, ESI $\dagger$ ), while the familiar salt-bridge and cation $-\pi$ interactions are present (Fig. S8a, b and e, ESI $\dagger$ ). The anion $-\pi$ (Fig. S8c, ESI $\dagger$ ) and sulfur $-\pi$ (Fig. S8d, ESI $\dagger$ ) bond distances are approximately $3.0 \AA$ and $3.5 \AA$, respectively at the end of $10 \mathrm{~ns}$. However, we mainly focus on 7-Ga here because 6-Ga is synthetically unfeasible (it is isolated at $4 \%$ yield). ${ }^{49}$

We predict two interactions (salt-bridge and cation- $\pi$ ) between Lys58 and Lys 112 with 7-Ga (Fig. 8). The predicted binding energy (scored ucav energy) for 7-Ga is comparable to that of 4-Ga and 1-Ga (Table 2).

The results for the $10 \mathrm{~ns}$ of MD simulations are summarized in Fig. 9. We find that the docked binding mode is stable (Fig. S9, ESI $\dagger$ ), with all key interactions from the docking maintained during the MD (Fig. S10, ESI $\dagger$ and Fig. 10). Moreover we find the sulfur- $\pi$ interaction as observed in 1-Ga (Fig. 9b),

Table 2 Docking results of 7-Ga to Hsp90

\begin{tabular}{llllll}
\hline Ligand-Hsp90 complex & Snapbe $\left(\mathrm{kcal} \mathrm{mol}^{-1}\right)$ & Scored ucav $\left(\mathrm{kcal} \mathrm{mol}^{-1}\right)$ & Type of interaction & Interaction & Bond length $(\AA)$ \\
\hline 7-Ga & -145.08 & -124.50 & Salt-bridge & (Lys112)NZ-O58 & 2.9 \\
& & & Cation- $\pi$ & (Lys58)NZ-C28 & 4.0 \\
& & & Anion- $\pi$ & (Asp102)OD2-C59 & 4.9
\end{tabular}
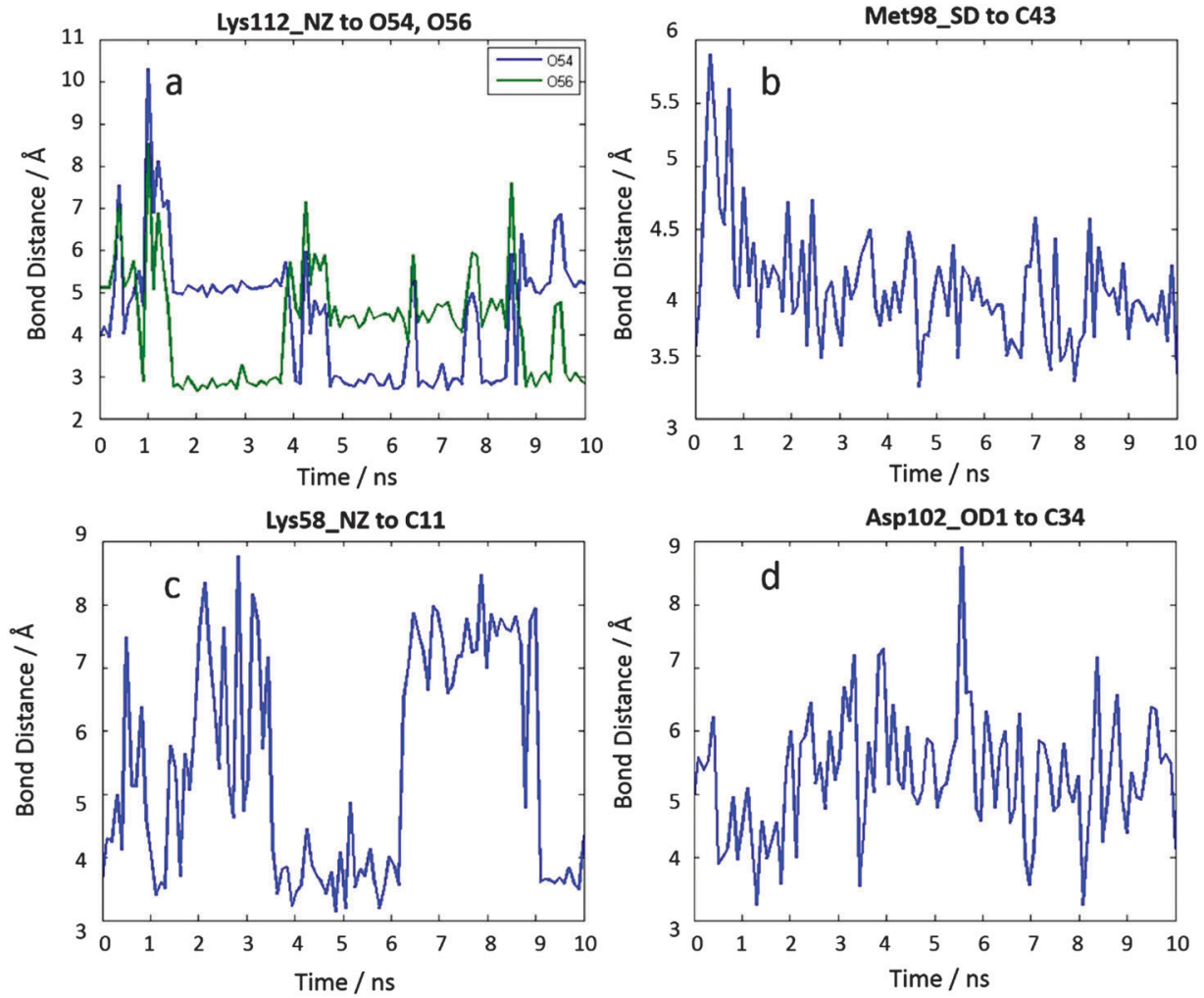

Fig. $910 \mathrm{~ns}$ MD results of the interaction distances of 7-Ga to Hsp90 starting from the docking pose c23708. (a) Lys112 NZ to O54, Lys112 NZ to O56, (b) Met98 SD to C43, (c) Lys58 NZ to C11, (d) Asp102 OD1 to C34. 


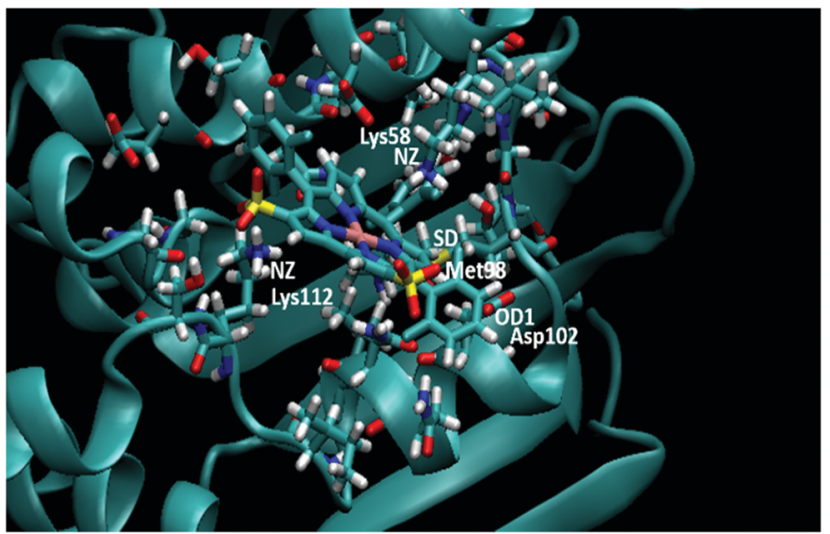

Fig. 10 Ending frame of 7-Ga to Hsp90 after 10 ns MD. The starting pose is shown in Fig. S10 (ESI $\dagger$ ). Relevant residues are highlighted.

although both the sulfur $-\pi$ and anion $-\pi$ bond distances are about $1 \AA$ A longer than that observed in 6-Ga (Fig. S8, ESI $\dagger$ ).

\section{Conclusions}

Our docking and MD simulation results suggest that the experimentally observed cytotoxicity of 1-Ga might be the result of its inhibition of Hsp90. We also propose a new gallium corrole derivative, 7-Ga, as a potential Hsp90 inhibitor, suggesting it as a promising candidate for further experimental investigation. These studies suggest a new understanding of the tumor-killing mechanism of metallocorroles, and we have provided a pharmacophore that can help develop this new class of Hsp90 inhibitors.

\section{Acknowledgements}

A Caltech/COH grant (H.B.G. and Z.G.) and the AACR-Thomas J. Bardos Science Education Award (R.D.T.) are gratefully acknowledged. SSD and WAG were supported partially by NIH (R01NS073115 and R01AI040567). We would also like to thank Y. C. Lam for helpful discussions.

\section{Notes and references}

1 Z. Okun, L. Kupershmidt, T. Amit, S. Mandel, O. Bar-Am, M. B. H. Youdim and Z. Gross, ACS Chem. Biol., 2009, 4, 910.

2 L. Kupershmidt, Z. Okun, T. Amit, S. Mandel, I. Saltsman, A. Mahammed, O. Bar-Am, Z. Gross and M. B. H. Youdim, J. Neurochem., 2010, 113, 363.

3 A. Kanamori, M. M. Catrinescu, A. Mahammed, Z. Gross and L. A. Levin, J. Neurochem., 2010, 114, 488.

4 A. Haber, A. Mahammed, B. Fuhrman, N. Volkova, R. Coleman, T. Hayek, M. Aviram and Z. Gross, Angew. Chem., Int. Ed., 2008, 47, 7896.

5 A. Preuß, I. Saltsman, A. Mahammed, M. Pfitzner, I. Goldberg, Z. Gross and B. Röder, J. Photochem. Photobiol., B, 2014, 133, 39.

6 D. Kowalska, X. Liu, U. Tripathy, A. Mahammed, Z. Gross, S. Hirayama and R. P. Steer, Inorg. Chem., 2009, 48, 2670.
7 R. D. Teo, H. B. Gray, P. Lim, J. Termini, E. Domeshek and Z. Gross, Chem. Commun., 2014, 50, 13789.

8 A. Mahammed, H. B. Gray, J. J. Weaver, K. Sorasaenee and Z. Gross, Bioconjugate Chem., 2004, 15, 738.

9 A. Haber, H. Agadjanian, L. K. Medina-Kauwe and Z. Gross, J. Inorg. Biochem., 2008, 102, 446.

10 A. Haber, M. Aviram and Z. Gross, Chem. Sci., 2011, 2, 295.

11 H. Agadjanian, J. J. Weaver, A. Mahammed, A. Rentsendorj, S. Bass, J. Kim, I. J. Dmochowski, R. Margalit, H. B. Gray, Z. Gross and L. K. Medina-Kauwe, Pharm. Res., 2006, 23, 367.

12 H. Agadjanian, J. Ma, A. Rentsendorj, V. Valluripalli, J. Y. Hwang, A. Mahammed, D. L. Farkas, H. B. Gray, Z. Gross and L. K. Medina-Kauwe, Proc. Natl. Acad. Sci. U. S. A., 2009, 106, 6105.

13 A. Mahammed and Z. Gross, J. Am. Chem. Soc., 2005, 127, 2883. 14 I. Saltsman, A. Mahammed, I. Goldberg, E. Tkachenko, M. Botoshansky and Z. Gross, J. Am. Chem. Soc., 2002, 124, 7411. 15 A. Mahammed and Z. Gross, J. Inorg. Biochem., 2002, 88, 305. 16 J. Y. Hwang, J. Lubow, D. Chu, J. Ma, H. Agadjanian, J. Sims, H. B. Gray, Z. Gross, D. L. Farkas and L. K. Medina-Kauwe, Mol. Pharmaceutics, 2011, 8, 2233.

17 J. Y. Hwang, D. J. Lubow, D. Chu, J. Sims, F. AlonsoValenteen, H. B. Gray, Z. Gross, D. L. Farkas and L. K. Medina-Kauwe, J. Controlled Release, 2012, 163, 368.

18 H. Charlotte, Nat. Rev. Drug Discovery, 2013, 12, 346.

19 H. Yamaki, M. Nakajima, K. W. Shimotohno and J. Tanaka, Antibiotics, 2011, 64, 635.

20 R. Bhat, S. R. Tummalapalli and D. P. Rotella, J. Med. Chem., 2014, 57, 8718.

21 D. S. Hong, U. Banerji, B. Tavana, G. C. George, J. Aaron and R. Kurzrock, Cancer Treat. Rev., 2013, 39, 375.

22 S. Paul and S. Mahanta, Mol. Cell. Biochem., 2014, 386, 45. 23 L. Neckers and P. Workman, Clin. Cancer Res., 2012, 18, 64. 24 R. R. A. Kitson and C. J. Moody, J. Org. Chem., 2013, 78, 5117. 25 B. L. Dutton, R. R. A. Kitson, S. Parry-Morris, S. M. Roe, C. Prodromou and C. J. Moody, Org. Biomol. Chem., 2014, 12, 1328.

26 R. R. A. Kitson, C.-H. Chang, R. Xiong, H. E. L. Williams, A. L. Davis, W. Lewis, D. L. Dehn, D. Siegel, S. M. Roe, C. Prodromou, D. Ross and C. J. Moody, Nat. Chem., 2013, 5, 307.

27 W.-Y. Chen, F.-R. Chang, Z.-Y. Huang, J.-H. Chen, Y.-C. Wu and C.-C. Wu, J. Biol. Chem., 2008, 283, 17184.

28 P. Lim, A. Mahammed, Z. Okun, I. Saltsman, Z. Gross, H. B. Gray and J. Termini, Chem. Res. Toxicol., 2011, 25, 400. 29 W. H. Lee, J. M. Lee, C. Lim, S. Kim and S. G. Kim, Chem.-Biol. Interact., 2013, 204, 49.

30 K. E. Thomas, A. B. Alemayehu, J. Conradie, C. Beavers and A. Ghosh, Inorg. Chem., 2011, 50, 12844.

31 P. J. Hay and W. R. Wadt, J. Chem. Phys., 1985, 82, 270.

32 P. J. Hay and W. R. Wadt, J. Chem. Phys., 1985, 82, 284.

33 A. D. Bochevarov, E. Harder, T. F. Hughes, J. R. Greenwood, D. A. Braden, D. M. Philipp, D. Rinaldo, M. D. Halls, J. Zhang and R. A. Friesner, Int. J. Quantum Chem., 2013, 113, 2110.

34 W. A. Goddard, S.-K. Kim, Y. Li, B. Trzaskowski, A. R. Griffith and R. Abrol, J. Struct. Biol., 2010, 170, 10. 
35 W. B. Floriano, N. Vaidehi, G. Zamanakos and W. A. Goddard, J. Med. Chem., 2004, 47, 56.

36 S. L. Mayo, B. D. Olafson and W. A. Goddard, J. Phys. Chem., 1990, 94, 8897.

37 S. S. Dong, R. Abrol and W. A. Goddard, ChemMedChem, 2015, 10, 650.

38 N. Nair, W. Kudo, M. A. Smith, R. Abrol, W. A. Goddard and V. P. Reddy, Bioorg. Med. Chem. Lett., 2011, 21, 3957.

39 The PyMOL Molecular Graphics System, Version 1.7.4, Schrödinger, LLC.

40 C. E. Stebbins, A. A. Russo, C. Schneider, N. Rosen, F. U. Hartl and N. P. Pavletich, Cell, 1997, 89, 239.

41 G. Banumathy, V. Singh, S. R. Pavithra and U. Tatu, J. Biol. Chem., 2003, 278, 18336.

42 J. C. Phillips, R. Braun, W. Wang, J. Gumbart, E. Tajkhorshid, E. Villa, C. Chipot, R. D. Skeel, L. Kale and K. Schulten, J. Comput. Chem., 2005, 26, 1781.

43 W. Humphrey, A. Dalke and K. Schulten, J. Mol. Graphics, 1996, 14, 33.
44 D. A. Case, T. A. Darden, T. E. Cheatham III, C. L. Simmerling, J. Wang, R. E. Duke, R. Luo, R. C. Walker, W. Zhang, K. M. Merz, B. Roberts, B. Wang, S. Hayik, A. Roitberg, G. Seabra, I. Kolossváry, K. F. Wong, F. Paesani, J. Vanicek, J. Liu, X. Wu, S. R. Brozell, T. Steinbrecher, H. Gohlke, Q. Cai, X. Ye, J. Wang, M.-J. Hsieh, G. Cui, D. R. Roe, D. H. Mathews, M. G. Seetin, C. Sagui, V. Babin, T. Luchko, S. Gusarov, A. Kovalenko and P. A. Kollman, AMBER 11, University of California, San Francisco, 2010.

45 C. Zipp, PhD thesis, University of the Witwatersrand, 2014. 46 J. Wang, R. M. Wolf, J. W. Caldwell, P. A. Kollman and D. A. Case, J. Comput. Chem., 2004, 25, 1157.

47 A. K. Rappé, C. J. Casewit, K. Colwell, W. A. Goddard and W. Skiff, J. Am. Chem. Soc., 1992, 114, 10024.

48 J. M. Lee, W. H. Lee, H. Y. Kay, E.-S. Kim, A. Moon and S. G. Kim, Int. J. Cancer, 2012, 130, 716.

49 A. Mahammed and Z. Gross, J. Porphyrins Phthalocyanines, 2010, 14, 911. 